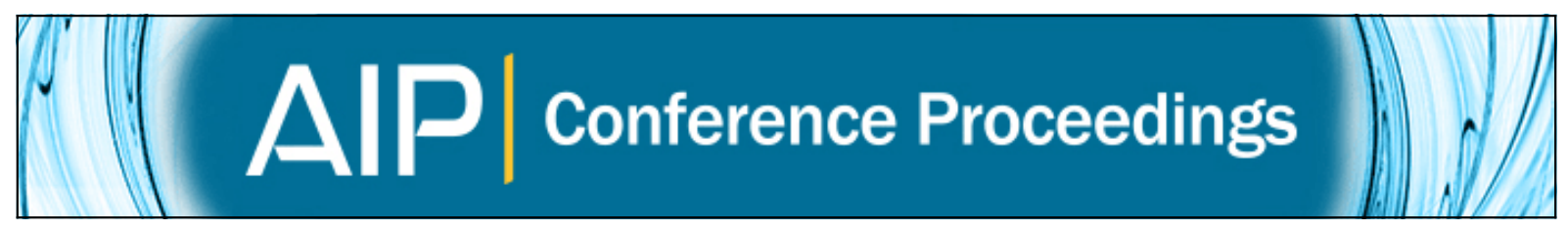

\title{
Signatures of doubly-charged Higgsinos at colliders
}

D. A. Demir, M. Frank, K. Huitu, S. K. Rai, and I. Turan

Citation: AIP Conference Proceedings 1078, 312 (2008); doi: 10.1063/1.3051945

View online: http://dx.doi.org/10.1063/1.3051945

View Table of Contents: http://scitation.aip.org/content/aip/proceeding/aipcp/1078?ver=pdfcov

Published by the AIP Publishing

\section{Articles you may be interested in}

How To Determine SUSY Mass Scales Now

AIP Conf. Proc. 1078, 57 (2008); 10.1063/1.3052033

(SUSY) Higgs Search at the LHC

AIP Conf. Proc. 1078, 30 (2008); 10.1063/1.3051944

Signatures of non-universal gaugino and scalar masses at the Large Hadron Collider (LHC)

AIP Conf. Proc. 1078, 277 (2008); 10.1063/1.3051933

Top quark and charged Higgs production at hadron colliders

AIP Conf. Proc. 792, 643 (2005); 10.1063/1.2122120

Multi-Lepton Events at $\mathrm{H} 1$ and Search for Doubly-Charged Higgs Bosons

AIP Conf. Proc. 792, 615 (2005); 10.1063/1.2122113 


\title{
Signatures of doubly-charged Higgsinos at colliders
}

\author{
D. A. Demir*,ं, M. Frank**, K. Huitu ${ }^{*}$, S. K. Rai and I. Turan** \\ *Department of Physics, Izmir Institute of Technology, IZTECH, TR35430 Izmir, Turkey. \\ ${ }^{\dagger}$ Deutsches Elektronen - Synchrotron, DESY, D-22603 Hamburg, Germany. \\ ${ }^{* *}$ Department of Physics, Concordia University, 7141 Sherbrooke Street West, Montreal, Quebec, CANADA H4B \\ $1 R 6$. \\ ${ }^{\ddagger}$ Department of Physics, University of Helsinki and Helsinki Institute of Physics, P.O. Box 64, FIN-00014, \\ Helsink, Finland
}

\begin{abstract}
Several supersymmetric models with extended gauge structures predict light doubly-charged Higgsinos. Their distinetive signature at the large hadron collider is highlighted by studying its production and decay characteristics.
\end{abstract}

Keywords: left-right supersymmetry, doubly charged higgsinos.

PACS: $12.60 . \mathrm{Jv}, 12.60 . \mathrm{Fr}$

\section{INTRODUCTION}

Supersymmetry (SUSY), in the form of the minimal supersymmetric standard model (MSSM) is the most popular scenario of physics beyond the standard model (SM) and resolves the gauge hierarchy problem very elegantly. A general, although not universally present, feature of SUSY, is that, if R-parity $R=(-1)^{(3 B+L+2 S)}$ (with $B$, $L$ and $S$ being baryon, lepton and spin quantum numbers, respectively) is conserved, the absolute stability of the lightest supersymmetric partner (LSP) is guaranteed making it a viable candidate for cold dark matter in the universe. However to generate neutrino masses, one must either invoke R-parity violation and abandon the absolute stability of LSP or add right-handed neutrinos and introduce the seesaw mechanism. Supersymmetric grand unified theories (SUSY GUTs) which contain left-right supersymmetry (LRSUSY) resolve both problems naturally $[1,2]$. The LRSUSY gauge theory is based on the product group $S U(3)_{C} \times S U(2)_{L} \times S U(2)_{R} \times U(1)_{B-L}$. LRSUSY models are attractive for many reasons. They disallow explicit R-parity breaking in the Lagrangian; they provide a natural mechanism for generating neutrino masses; and they provide a solution to the strong and weak CP problem in MSSM [3]. Neutrino masses are induced by the see-saw mechanism through the introduction of Higgs triplet fields which transform as the adjoint of the $S U(2)_{R}$ group and have quantum numbers $B-L= \pm 2$. While the Higgs triplet bosons are present in the non-supersymmetric version of the theory, their fermionic partners, the higgsinos, are specific to the supersymmetric version. It has been shown that, if the scale for left-right symmetry breaking is chosen so that the light neutrinos have the experimentally expected masses, these higgsinos can be light, with masses in the range of $\mathscr{O}(100) \mathrm{GeV}$ [4]. Such particles could be produced in abundance and thus give definite signs of left-right symmetry at future colliders like the LHC and linear $e^{+} e^{-}$colliders. For a more detailed information about the model see, for instance $[1,5]$.

As the underlying theory to model "new physics" at the $\mathrm{TeV}$ scale bring about new particles and interaction schemes, experiments at the Large Hadron Collider (LHC) will be probing these new particles as well as new interactions. The models of new physics will be distinguished by certain characteristic signatures in regard to their lepton and jet spectra in the final state. In this talk we present signatures specific to the LRSUSY model at the LHC. We consider the pair production and single production of the doubly charged Higgsinos at LHC and analyze the signals resulting from its decays. We refer the readers to $[6,7]$ for a more detailed account on the analysis.

\section{Production and decay of doubly charged Higgsinos}

The pair-production process at the LHC is $p p \longrightarrow$ $\widetilde{\Delta}^{++} \widetilde{\Delta}^{--}$which proceeds with $s$-channel $\gamma$ and $Z_{L, R}$ exchanges, and the associated production mode $p p \longrightarrow$ $\widetilde{\chi}_{1}^{+} \widetilde{\Delta}^{--}$which rests on $s$-channel $W_{L, R}$ exchanges. Both processes are generated by quark-anti-quark annihilation at the parton level. These doubly- and singly-charged fermions subsequently decay via a chain of cascades until the lightest neutralino $\chi_{1}^{0}$ is reached. In general, the two-body decays of doubly-charged Higgsinos are given by

$$
\cdot \widetilde{\Delta}^{--} \longrightarrow \widetilde{\ell}^{-} \ell^{-}, \Delta^{--} \widetilde{\chi}_{i}^{0}, \widetilde{\chi}_{i}^{-} \Delta^{-}, \widetilde{\chi}_{i}^{-} W^{-}
$$


TABLE 1. The low lying mass spectrum in the model, defining the sample points SPA, SPB and SPC. In each case, $\mathbf{S} \mathbf{2}$ and $\mathbf{S} \mathbf{3}$ designate parameter values which allow for 2-body and 3-body decays of doubly-charged Higgsinos, respectively.

\begin{tabular}{|c|c|c|c|}
\hline fields ${ }^{*}$ & SPA & SPB & SPC \\
\hline$\widetilde{\chi}_{i}^{0}(i=1,3)$ & $89.9,180.6,250.9$ & $212.9,441.2,458.5$ & $142.5,265.6,300.0$ \\
\hline$\widetilde{\chi}_{i}^{ \pm}(i=1,3)$ & $250.9,300.0,953.9$ & $459.4,500.0,500.0$ & $300.0,459.3,500.0$ \\
\hline$M_{\widetilde{\Delta}}$ & 300 & 500 & 300 \\
\hline$W_{R}^{\Delta}, Z_{R}$ & $2090.4,3508.5$ & $1927.2,3234.8$ & $1927.2,3234.8$ \\
\hline & S2 $\quad$ S3 & S3 & S3 \\
\hline$\widetilde{e}_{L}, \widetilde{e}_{R}$ & $(156.9,155.6),(402,402)$ & $(254.2,253.4),(552,552)$ & $(214.9,214.0),(402.6,402.2)$ \\
\hline$\widetilde{\mu}_{L}, \widetilde{\mu}_{R}$ & $(156.9,155.6),(402,402)$ & $(254.2,253.4),(552,552)$ & $(214.9,214.0),(402.6,402.2)$ \\
\hline$\widetilde{\tau}_{1}, \widetilde{\tau}_{2}$ & $(155.4,159.9),(401,406)$ & $(252.5,257.9),(550,556)$ & $(212.8,216.2),(401.5,403.3)$ \\
\hline
\end{tabular}

* all masses in GeV

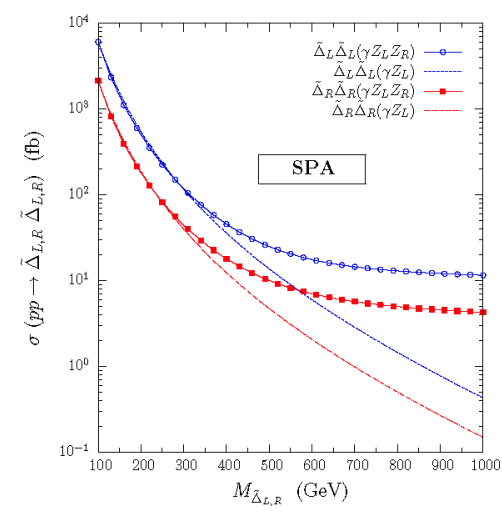

FIGURE 1. The pair-production cross sections for doublycharged Higgsinos in LRSUSY at the LHC.

whose decay products further cascade into lower-mass daughter particles of which leptons are of particular interest.

We choose three sample points in the LRSUSY parameter space, as tabulated in Table 1. A quick look at the mass spectrum for the sparticles suggest that the chargino states are also heavier than or comparable to the doublycharged Higgsinos, and hence, the favorable decay channel for $\widetilde{\Delta}$ is $\widetilde{\Delta}^{--} \longrightarrow \widetilde{\ell}^{-} \ell^{-}$(S2), provided that $m_{\tilde{l}}<$ $M_{\widetilde{\Lambda}^{--}}$. For relatively light Higgsinos, one can, in principle, have $m_{\tilde{l}}>M_{\tilde{\Delta}^{--}}$in which case the only allowed decay mode for the doubly-charged Higgsinos would be the 3-body decays, which would proceed dominantly through off-shell sleptons: $\widetilde{\Delta}^{--} \rightarrow \widetilde{\ell}^{\star-} \ell^{-} \rightarrow \ell^{-} \ell^{-} \widetilde{\chi}_{1}^{0}$ (S3).

For the benchmark point in Table 1, the doublycharged Higgsinos assume the following 2- and 3-body decay branchings:

$$
\begin{aligned}
B R\left(\widetilde{\Delta}_{L / R}^{--} \rightarrow \tilde{\ell}_{i L / i R}^{-} \ell_{i}^{-}\right) & \simeq \frac{1}{3}, m_{\tilde{l}_{i}}<M_{\tilde{\Delta}^{--}} \\
B R\left(\tilde{\ell}_{i L / i R}^{-} \rightarrow \ell_{i}^{-} \tilde{\chi}_{1}^{0}\right) & =1,
\end{aligned}
$$

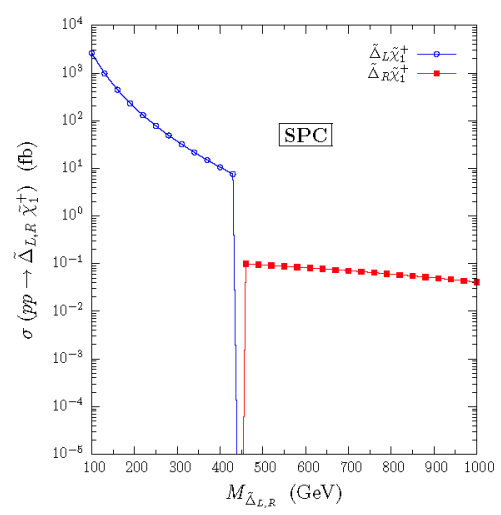

FIGURE 2. The cross sections for associated productions of $\widetilde{\Delta}_{L, R}$ and $\tilde{\chi}_{1}^{ \pm}$in the LRSUSY model at LHC.

$$
B R\left(\widetilde{\Delta}_{L / R}^{--} \rightarrow \ell_{i}^{-} \ell_{i}^{-} \widetilde{\chi}_{1}^{0}\right) \simeq \frac{1}{3}, m_{\tilde{l}_{i}}>M_{\tilde{\Delta}^{--}}
$$

where $i=e, \mu, \tau$.

The pair-production and associated production cross sections are shown in Figs 1,2. The doubly-charged Higgsinos decay according to (1) into two same-sign sameflavor (SSSF) leptons and the lightest neutralino $\widetilde{\chi}_{1}^{0}$, the LSP. This decay pattern, when the doubly charged states are produced in pair, give rise to final states involving four isolated leptons of the form

$$
p p \longrightarrow \tilde{\Delta}^{++} \tilde{\Delta}^{--} \longrightarrow\left(\ell_{i}^{+} \ell_{i}^{+}\right)+\left(\ell_{j}^{-} \ell_{J}^{-}\right)+H_{T},
$$

where $\ell_{i}, \ell_{j}=e, \mu, \tau$. Similarly, in the case of associated production one gets

$$
p p \longrightarrow \widetilde{\Delta}^{--} \tilde{\chi}_{1}^{+} \longrightarrow\left(\ell_{i}^{-} \ell_{i}^{-}\right)+\ell_{j}^{+}+Z_{T},
$$

The $4 \ell+E_{T}$ signal receives contributions from the pair-production of both chiral states of the doublycharged Higgsino so we add up their individual contributions to obtain the total number of events. This yields a 

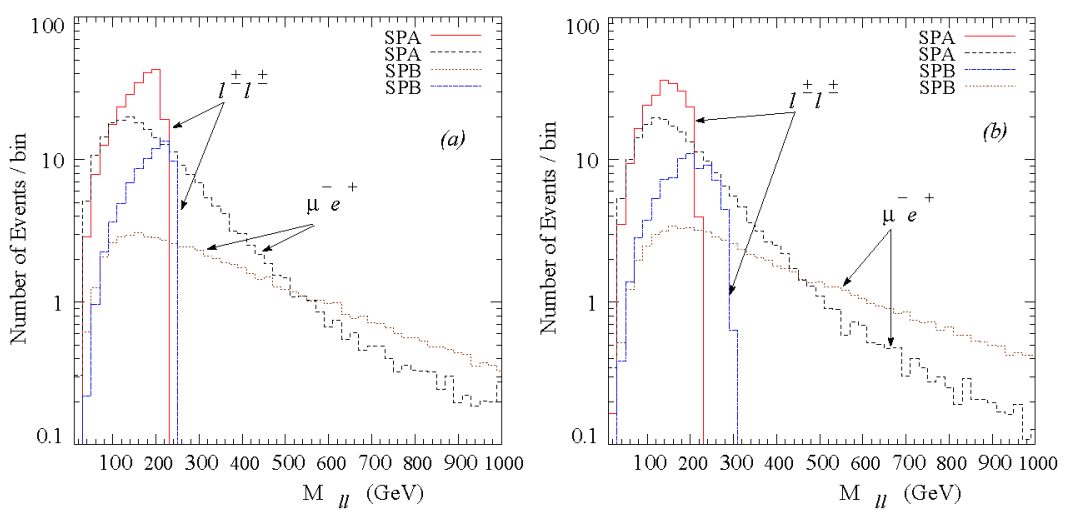

FIGURE 3. Binwise invariant mass distribution of lepton pairs with integrated luminosity of $\int \mathscr{L} d t=30 f b^{-1}$. The panel (a) represents the 2-body (S2) case, and panel (b) does the 3-body (S3) case.

rather clean and robust $4 \ell+$ missing $p_{T}$ signal at the LHC with highly suppressed SM background. We impose the following kinematic cuts on our final states:

- The charged leptons in the final state satisfy $\left|\eta_{\ell}\right|<$ 2.5 , and have a minimum transverse momentum $p_{T}>25 \mathrm{GeV}$. Each pair of oppositely-charged leptons of same flavor have at least $10 \mathrm{GeV}$ invariant mass.

- To ensure proper resolution between the final state leptons we demand $\Delta R_{\ell \ell}>0.4$ for each pair of leptons.

- The missing transverse energy must be $F_{T}>50$ $\mathrm{GeV}$.

The total cross section for the $2 \mu^{-}+2 e^{+}+H_{T}$ signal for SPA point is $7.71 \mathrm{fb}(\mathbf{S 2})$ and $7.02 \mathrm{fb}(\mathbf{S 3})$ while for SPB it is $2.43 \mathrm{fb}(\mathbf{S 2})$ and $2.66 \mathrm{fb}(\mathbf{S 3})$. In the case where the doubly charged Higgsino is produced in association with a chargino, we have used the SPC point, and the corresponding cross sections for $2 \mu^{-}+e^{+}+\#_{T}$ are $2.24 \mathrm{fb}(\mathbf{S 2})$ and $2.03 \mathrm{fb}(\mathbf{S 3})$ respectively. As in pair-production, the $\widetilde{\Delta}^{--}$decays again into a pair of SSSF leptons and an LSP, while the chargino decays with almost $100 \%$ branching ratio to a neutrino and slepton for SPC. In Fig. 3 we show the binwise invariant mass distribution of leptons pairs for both SPA,SPB points and both cases of S2 and S3. These plots manifestly show differences between the SSSF and opposite-signdifferent-flavor (OSDF) lepton pairs in regard to their invariant mass distributions. Indeed, the SSSF lepton pairs exhibit a sharp kinematic edge according to the expression given by

$$
M_{\ell^{ \pm} \ell^{ \pm}}^{\max }=\sqrt{M_{\widetilde{\Delta}}^{2}+M_{\widetilde{\chi}_{1}^{0}}^{2}-2 M_{\widetilde{\Delta}^{-} E_{\widetilde{\chi}_{1}^{0}}}}
$$

where $E_{\widetilde{\chi}_{1}^{0}}$ is the energy of the LSP, in their $M_{\ell \ell}$ distributions whereas the OSDF lepton pairs do not. These act as very effective discriminants when compared to similar signals in MSSM. The edge in the SSSF dilepton invariant mass distribution yields a clear hint of a $\Delta L=2$ interaction and a doubly-charged field in the underlying model of 'new physics'. A very similar feature is expected for SPC point and the $3 \ell+Z_{T}$ signal. The invariant mass distribution and also the distribution in $\Delta R$ of a pair SSSF and OSDF leptons, prove to be a very effective discriminant when compared with similar signals coming from other new physics scenarios. We refer the readers to [7] for further details.

\section{REFERENCES}

1. M. Cvetic and J. Pati, Phys. Lett. B 135, 57 (1984); R. N. Mohapatra and A. Rasin, Phys. Rev. D 54, 5835 (1996); R. Kuchimanchi, Phys. Rev. Lett. 76, 3486 (1996); R. N. Mohapatra, A. Rašin and G. Senjanović, Phys. Rev. Lett. 79, 4744 (1997); C. S. Aulakh, K. Benakli and G. Senjanovic, Phys. Rev Lett. 79, 2188 (1997); C. S. Aulakh, A. Melfo and G. Senjanovic, Phys. Rev D 57, 4174 (1998).

2. D. A. Demir, M. Frank and I. Turan, Phys. Rev. D $\mathbf{7 3}$, 115001 (2006).

3. R. N. Mohapatra and A. Rasin, Phys. Rev. Lett. 76, 3490 (1996); R. N. Mohapatra and A. Rasin, Phys. Rev. D 54, 5835 (1996); R. Kuchimanchi, Phys. Rev. Lett. 76, 3486 (1996).

4. Z. Chacko and R. N. Mohapatra, Phys. Rev. D 58, 015003 (1998); B. Dutta and R. N. Mohapatra, Phys. Rev. D 59, 015018 (1999).

5. R. M. Francis, M. Frank and C. S. Kalman, Phys. Rev. D 43, 2369 (1991); K. Huitu, J. Maalampi and M. Raidal, Phys. Lett. B 328, 60 (1994); K. Huitu, J. Maalampi and M. Raidal, Nucl. Phys. B420, 449 (1994); K. Huitu and J. Maalampi, Phys. Lett. B 344, 217 (1995).

6. M. Frank, K. Huitu and S. K. Rai, Phys. Rev. D 77, 015006 (2008).

7. D. A. Demir, M. Frank, K. Huitu, S. K. Rai and I. Turan, Phys. Rev. D 78, 035013 (2008). 